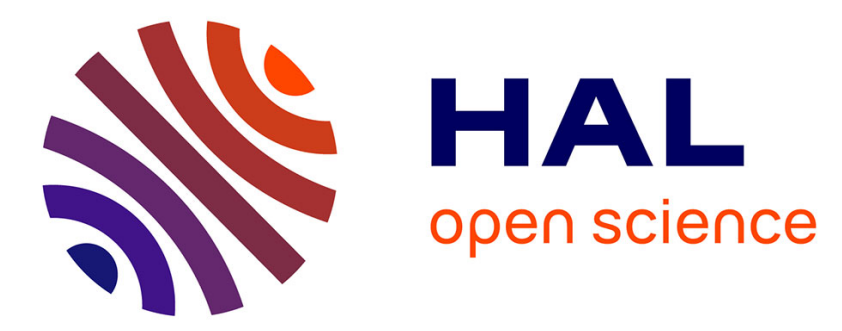

\title{
PHM-oriented Degradation Indicators for Batteries and Fuel Cells
}

\author{
Dacheng Zhang, Catherine Cadet, Nadia Yousfi-Steiner, Florence Druart, \\ Christophe Bérenguer
}

\section{- To cite this version:}

Dacheng Zhang, Catherine Cadet, Nadia Yousfi-Steiner, Florence Druart, Christophe Bérenguer. PHM-oriented Degradation Indicators for Batteries and Fuel Cells. Fuel Cells, 2017, Special Issue: 6th International Conference on Fundamentals \& Developments of Fuel Cells (FDFC2015), 3rd - 5th February 2015, Toulouse, France, 17 (2), pp.268-276. 10.1002/fuce.201600075 . hal-01505762

\section{HAL Id: hal-01505762 \\ https://hal.science/hal-01505762}

Submitted on 11 Apr 2017

HAL is a multi-disciplinary open access archive for the deposit and dissemination of scientific research documents, whether they are published or not. The documents may come from teaching and research institutions in France or abroad, or from public or private research centers.
L'archive ouverte pluridisciplinaire HAL, est destinée au dépôt et à la diffusion de documents scientifiques de niveau recherche, publiés ou non, émanant des établissements d'enseignement et de recherche français ou étrangers, des laboratoires publics ou privés. 


\title{
PHM-oriented Degradation Indicators for Batteries and Fuel
}

\section{Cells}

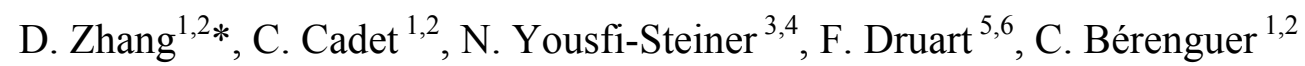

${ }^{1}$ Univ. Grenoble Alpes, GIPSA-Lab, F-38000 Grenoble

${ }^{2}$ CNRS, GIPSA-Lab, F-38000 Grenoble, France

${ }^{3}$ Univ. Bourgogne Franche-Comté, F-90010 Belfort, France

${ }^{4}$ FCLAB, FR CNRS 3539, FEMTO-ST, UMR CNRS 6174, Labex ACTION CNRS, Rue

Thierry Mieg, 90010 Belfort Cedex, France

${ }^{5}$ Univ. Grenoble Alpes, LEPMI, F-38000 Grenoble, France

${ }^{6}$ CNRS, LEPMI, F-38000 Grenoble, France

[*]Corresponding author: dacheng.zhang@gipsa-lab.grenoble-inp.fr

\begin{abstract}
As electrochemical power sources technologies, namely Li-ion batteries and PEM fuel cells, continue advancing, ways to extend their lifespan have to be pursued. With Prognostics and Health Management (PHM) approach, several techniques can provide estimation of State of Health (SOH) and prediction of Remaining Useful Life (RUL) in order to help the manufacturers improving performance. Before developing a solution, PHM issues need to be properly defined, in particular in terms of deterioration/ageing indicators used for $\mathrm{SOH}$ estimation and RUL prediction.

This paper reviews the ageing phenomena of batteries and fuel cells in order to investigate how to define suitable degradation indicators for these devices. Since Li-ion battery' PHM is much more developed, their similarities and differences have thus been studied as an exploration path of how Proton Exchange Membrane fuel cell (PEMFC) could benefit from the battery knowledge and experience feedback. Even if no innovative solutions are emerging,
\end{abstract}


the relevance of the covariates used for PEMFC and batteries ageing indicators are argued, providing a first step to build a suitable PHM approach for electrochemical devices.

Keywords: PEM Fuel Cell, Battery, PHM, State Of Health, degradation indicator.

\section{Introduction}

Presently, the spotlight is on fuel cell systems among energy conversion devices. Even if this technology is close to being competitive, it is not yet ready to be considered for a large-scale industrial deployment. Proton Exchange Membrane, also known as Polymer Electrolyte Membrane fuel cells, are considered a possible answer to environmental and energy problems. They have been considered as one of the most promising technologies for both stationary and transportation applications among different types of fuel cells [1]. However, the PEMFC has not been as thoroughly studied as lithium ion battery, which is by far the most popular in the worldwide in electric mobility applications $[2,3]$, The durability and the reliability are the most critical challenges to be faced. Thus, studying the degradation behavior of fuel cell systems and Li-ion batteries will lead to a better understanding of these technologies, in order to extend systems life span and to enhance their performance.

Prognostics and Health Management (PHM) is a recent dynamic approach to extend and manage the life duration of industrial systems. This technique has been relatively studied for batteries but it is pioneering in fuel cells technologies [4], unlike the diagnosis methods that have been widely explored [5-8]. The key process of PHM is prognosis. The prognosis can take into account the inherent uncertainty of failure phenomena to estimate the evolution of the state of health of a system, and thus provide its residual lifetime or Remaining Useful Life (RUL). Several indicators can be used to estimate the ageing process by quantifying the health level, called the State of Health (SOH). An ageing indicator should be representative of the 
degradation of the device and easy to measure, regarding the constraints linked to the targeted application.

In a fuel cell, the ageing process modifies both the performance and components materials. The degradation factors (or "stress factors") are determined by the manner in which the fuel cell is used, and are partly identified by diagnostics studies [9]. However, PEMFC stacks degradation phenomena are still far from being well understood. PEMFC ageing studies are very scarce and experimentations are long and costly, limiting strongly the possibilities [4]. Unlike for fuel cells, the battery prognosis has been studied for many years, and the experiments are much less expensive and much easier to achieve. The RUL for batteries is usually the remaining time or the number of load cycles until the battery reaches its end of life [2]. The battery SOC can be employed in the figurative sense as a replacement for a fuel gauge used in conventional vehicles. The SOC is basically the relationship between the residual battery capacity in its present state $\left(\mathrm{Q}_{\text {available }}\right)$ and total capacity $\mathrm{Q}$ after completely charging the battery, expressed in a percentage $(0 \%=$ empty; $100 \%=$ full $)$ :

$\operatorname{SOC}(t)=\frac{\text { Qavailable }(t)}{Q(t)}$

Most of the battery-state estimation methods were developed for either SOC or SOH estimation, not both. As the intimate coupling feature between SOC and SOH was overlooked [10], this paper have explored both estimation methods.

Batteries and Fuel cells are both electrochemical devices with structural similarities and thus some knowledge on batteries can possibly be transferred to PEMFCs. This paper reviews the ageing phenomena of batteries and fuel cells and investigates how to define suitable degradation indicators for these devices. The similarities and differences between Li-Ion batteries and PEMFC are studied with the aim to investigate how PEMFC could benefit from the experience feedback and cumulated knowledge in the field of Li-ion battery' PHM, which is much more developed. Even if no innovative solutions are emerging, the relevance of the 
covariates used for PEMFCs and batteries ageing indicators are argued, providing a first step to build a suitable PHM approach for electrochemical devices.

This paper is organised as follows: the following section 2 deals with operation and the ageing mechanisms for the two devices. Then, section 3 presents the tools for batteries and PEMFCs characterisation. In section 4, the measurements of the battery degradations are presented and the possibility to use similar measurements for PEMFC is discussed. Finally, the last part presents a selection of relevant covariates for batteries and PEMFC ageing indicators.

\section{Operation and ageing of batteries and PEMFC}

\subsection{Batteries and fuel cells: electrochemical cells}

A Li-ion battery consists of several elementary electrochemical cells that are electrically connected in series (increasing the maximum voltage) or in parallel (increasing the maximum current). An electrochemical cell, presented in Figure 1, comprises a negative electrode where oxidation reaction occurs and releases electrons as the cell discharges, and a positive electrode where reduction occurs. A stable electrolyte with required high ionic conductivity enables ion transfer between the two electrodes. This process reverses when the cell is at charging: the positive electrode takes the role of oxidation and the negative takes the role of reduction.

The electrodes consist of composite and porous materials: a mixture of a conductive carbonaceous with a polymer ensuring a good mechanical property. For example, for a battery with a positive electrode of lithium Cobalt oxide, the two electrochemical reactions at discharging are:

Negative: $x \mathrm{LiC}_{6} \rightarrow x \mathrm{Li}^{+}+x \mathrm{e}^{-}+x \mathrm{C}_{6}$

Positive: $\mathrm{Li}_{1-x} \mathrm{CoO}_{2}+x \mathrm{Li}^{+}+x e^{-} \rightarrow \mathrm{LiCoO}_{2}$

The two electrodes are separated by a polymer porous membrane (separator). There is an interface between negative electrode and electrolyte, known as Solid Electrolyte Interphase 
(SEI). The SEI is naturally generated in the first cycle allowing only the passage of Li-ions to protect the negative electrode from possible corrosion and to protect the electrolyte from reduction.

Very similarly, a fuel cell stack is an assembly of several cells in series and produce electricity through an electrochemical reaction. However, fuel cells are continuously fed by gases.. The simultaneous two half-cell electrochemical and overall reactions for a PEMFC are:

Anode: $\quad H_{2} \rightarrow 2 H^{+}+2 e^{-}$

Cathode: $\quad \frac{1}{2} \mathrm{O}_{2}+2 \mathrm{H}^{+}+2 e^{-} \rightarrow \mathrm{H}_{2} \mathrm{O}$

Overall reaction: $\quad \frac{1}{2} \mathrm{O}_{2}+\mathrm{H}_{2} \rightarrow \mathrm{H}_{2} \mathrm{O}$

In a PEMFC (Figure 2), the core component of a PEMFC is the Membrane Electrode Assembly (MEA). A typical MEA is composed of: two Gas Diffusion Layers (GDLs), two Active Layers (ALs) and a Proton Exchange Membrane (PEM).

The GDL is a porous material composed of a dense array of carbon fibers that provides an electrically conductive pathway for current collection. It performs the following essential functions: pathway for reactant transport until the AL, medium that optimizes liquid water removal by using Polytetrafluoroethylene (PTFE), mechanical support to the MEA and protection of the catalyst layer from corrosion or erosion caused by flows. The polymer membrane is a thin layer of polymer with high ionic conductivity, which conducts protons from the anode to the cathode while preventing electron transport and the cross-over of hydrogen fuel and oxygen reactant. The AL is a porous structure composed of a network of catalyst nanoparticles of platinum and ionomer fragments. It is the layer where the electrochemical reactions take place. The reaction activity is quantified as active surface. Unlike the Li-ion battery whose two electrodes are made of different materials, PEMFC electrodes' materials are similar. Both of the positive (anode) and negative electrode (cathode) 
are formed by an AL and a GDL. The Electrochemically Active Surface Area cannot be measured directly but estimated in specific operating conditions.

In conclusion, batteries and PEMFC have a similar structure with a stack consisting of several elementary cells, each one composed of an assembly of electrode/electrolyte/electrode. In addition, the electrochemical principles of battery discharging and PEMFC are nearly the same. However the cells are of different type: PEMFC electrodes are porous with gas transport contrary to battery. The operating conditions are also fundamentally different. Battery is a batch process and its storage energy is limited by the active mass while PEMFC needs a constant supply of reactants, and water must be removed continuously. For PEMFC, power production is separated from energy storage which depends on external gas storage. The performance characterization will thus be different. For battery, voltage is plotted versus time whereas for PEMFC voltage is plotted versus current to give a polarization curve.

\subsection{Li-ion Battery Ageing Mechanism}

There are two modes of batteries ageing [2]. The first one is the calendar ageing which occurs while the battery is at rest: in an electrical vehicle; it is often known as "parking" mode. One should not confuse this type of ageing (irreversible) with the phenomenon of self-discharge (reversible). The calendar ageing mainly depends on two factors, the storage temperature and the battery SOC: the higher is the storage temperature, the faster is the calendar ageing. Similarly, a higher SOC level comes with more significant ageing. The second type of ageing, called "cycling ageing" occurs when the battery is either discharging or charging. The consequences may be similar to those recorded during a calendar ageing, but the causes are more complex with multiple operating conditions. Considering literature in this area, the most significant degradation factors in automotive application can be identified as environment temperature, discharging current rate, charging rate (fast charging), Depth Of Discharge (DOD) and time intervals between full charge cycles [11]. 
These ageing phenomena are numerals, complex and are difficult to investigate independently. The main ageing mechanisms can be categorized as follows:

(i) Negative electrode: changes at SEI due to reactions between the negative electrode and the electrolyte are considered to be the major source of ageing on Li-ion battery [12].

(ii) SEI: the growth and formation (composition) of SEI are continuous due to certain usage conditions such as overcharge and/or charge at low temperature or electrodes oxidation. They result in an increase of electrode impedance and electronic contact loss, which in turn leads to capacity fading.

(iii) High temperature and repeated large cycles cause electrolyte decomposition.

(iv) Positive electrode: low alteration within time depending on the chosen material [3]. Among these degradations, the degradation on the SEI layer is considered as the main factor influencing batteries ageing. It has been demonstrated [13] that active lithium loss due to electrolyte decomposition forming the solid electrolyte interphase (SEI) in the carbon negative is the dominant ageing mechanism for LiFePO4 batteries.

\subsection{PEMFC Ageing Mechanism}

PEMFC ageing modes are: calendar ageing under constant optimal conditions; start and stop cycles and inadequate operating conditions such as temperature, pressure and poor water management. Consequently, material degradations can be seen on each cell component [14]:

(i) Gas Diffusion Layers: changes in GDL properties such as decrease of conductivity and hydrophobicity loss.

(ii) Active Layers: the pure Pt catalyst may be contaminated by supply reactants [15]. The catalyst may lose activity due to sintering/migration of Pt particles on the carbon support, or detachment/dissolution of Pt into the electrolyte. It may also lose activity due to the corrosion of carbon support and thus the active surface decreases. 
(iii) Membrane: the mechanical, thermal, chemical and electrochemical mechanisms may cause pinhole formation, conductivity loss as well as fuel crossover.

A specific issue with fuel cells is that reversible damages can occur, which may be confused with ageing. For instance, incidents due to poor operating conditions that lead to flooding of the channels or the membrane, or drying out of the membrane are reversible when corrected rapidly and the fuel cell recovers its entire performance after correction. However, if these problems persist, they can lead to material degradation and ageing of the fuel cell. In short, numerous material degradations may impact the ageing of a PEMFC, whereas for batteries ageing the degradation of the SEI layer is considered as the main factor that influences batteries ageing. The external reasons that could cause material degradation and thus accelerate ageing (calendar or cycling) may be:

(i) For batteries: too high or too low external temperature.

(ii) For PEMFC: improper management of operating conditions (i.e. temperature, humidity, gas flow, pressure).

Though these strong differences, a common factor of ageing is the way to use the electrochemical device.

\section{Batteries and PEMFCs tools for characterization}

\subsection{Voltage measurement}

As any electrochemical cell, batteries and fuel cells obey to the same thermodynamic and kinetic laws. To express the thermodynamic voltage of an electrochemical cell $\left(\mathrm{E}_{\mathrm{th}}\right)$, the Nernst Equation can be written as:

$E_{t h}=E^{0}+R T / n F \ln \left(A_{p} / A_{R}\right)$

where $E^{0}$ is the electromotive force (emf) in standard conditions, $R$ is the gas constant, $A_{P}$ and $A_{R}$ are the activity product of the products and reactants, respectively. For an ideal cell, $E_{t h}$ corresponds to the open circuit voltage (OCV). 
When a current is drawn from the cell, three kinds of « overvoltage » appear: activation polarization $\eta_{\text {act }}$ (charge transfer at the interface electrode/electrolyte that slows down kinetics), ohmic losses $\eta_{O h m}$ (due to the electrical resistance of individual components and their contact) and concentration polarization $\eta_{\text {mass }}$ (due to mass transport limitation at the electrode/electrolyte interface).

For batteries and fuel cells [16], the voltage behavior is impacted by these polarizations when a current is drawn and the voltage E can be expressed as:

$E=O C V-\eta_{o h m}-\eta_{a c t}-\eta_{\text {mass }}$

These polarization effects affect the performance of these electrochemical generators. Then voltage measurement is usually carried out to characterize performance or cell capacity. Discharge curve (voltage versus time or SOC) are used for batteries while polarization curve (voltage versus current density) are used for fuel cells. Studies and fitting of these curves assess different influences/parameter, such as ageing, effect of temperature, rate of charge/discharge, membrane resistance (fuel cells), or cell capacity (batteries).

To obtain more detailed information on polarization effects, characterization under alternative current $(\mathrm{AC})$ is performed. This is Electrochemical Impedance Spectroscopy (EIS). Indeed EIS is an effective technique to separate the different contributors to performance of electrochemical devices. Only EIS will be developed in this paper, as it could be useful for PHM purposes.

\subsection{Electrochemical Impedance Spectroscopy}

\subsubsection{EIS: a dynamic characterization tool}

Electrochemical Impedance Spectroscopy (EIS) is a fruitful tool, used mainly to characterize dynamics of different phenomena and component materials of electrochemical generators. The principle of the EIS is the following: a sinusoidal low-amplitude current $I_{d y n}$ is applied on 
the device, which response in terms of voltage is $U_{d y n}$. The impedance of the electrochemical device is thus:

$Z=\frac{U_{d y n}}{I_{d y n}}$

For a given frequency range, calculation of the impedance $Z$ allows to construct an electrochemical impedance spectrum (Nyquist plot). For batteries, Figure 3 gives the shape of the Nyquist plot obtained in [17] in accordance with similar Nyquist plots found in the literature with a line (the Warburg line). For fuel cells, the sinusoidal current is added to a static current $I_{\text {stat }}$, and the corresponding part of the voltage is $U_{\text {stat. }}$ Figure 4 represents the Nyquist diagram for a PEMFC. It can be divided into four parts:

(i) An inductive part at the highest frequencies (above the $\mathrm{kHz}$ ), due to connection cables' inductance;

(ii) A purely resistive part, high-frequency resistance $R_{e}$ : around $1 \mathrm{kHz}$. Mainly due to the ionic resistance of the membrane.

(iii) For intermediate frequencies (usually between $1 \mathrm{~Hz}$ and $1 \mathrm{kHz}$ ), a capacitive loop $\left(C_{d l}\right)$ which is due to the accumulation of charges at the electrode-electrolyte interface and to the resistance of transfer of the electrons; i.e. charge transfer. The electrochemical reaction with the fastest kinetics is usually preponderant;

(iv) A diffusional part at lowest frequencies $(<1 \mathrm{~Hz})$ due to diffusion of species (reactants and products), i.e. the mass transfer $\left(Z_{w}\right)$. This part is dominated by the electrochemical reaction with the slowest kinetics.

EIS curves for FC and batteries show similar shapes of ideal electrochemical cell.

Nevertheless specific shapes appears for the low frequencies: the battery has a $45^{\circ}$ slope (Warburg line) whereas the PEMFC shows a second semi-circle. Depending on the usage history of the cell, additional semi-circles can be observed. For batteries, for instance, these 
semicircles originate from the solid electrolyte interface (SEI) layer and the electronic properties of the materials.

\subsubsection{Equivalent Circuit Model}

Equivalent Circuit Models (ECMs) are commonly used to represent the ageing phenomena or default of operation [18]. The general ECM for electrochemical generators is shown in Figure 5. The parameters are: $R_{e}$, the ohmic resistance for high frequency; $R_{c t}$, the charge transfer (polarization) resistance, $C_{d l}$, the double layer capacitor and $Z_{w}$, the non-linear Warburg impedance. PHM could use those parameters as they undergo variation with cell degradation and ageing.

For batteries, the double layer capacitor $C_{d l}$ represents the result of the variation of electric potential at the electrode/electrolyte interface, characterizes the charge accumulation phenomena in the double layer. The non-linear Warburg impedance $Z_{w}$ represents the diffusion phenomena. For PEMFC, the resistance of membrane is modelled as ohmic resistance $R_{e}$. Thus the pinhole formation or the conductivity loss at PEMFC due to ageing could reflect on the variation of $R_{e}$. To describe the effects of the electrodes polarization, the electrode impedance is considered taking into account both the activation and the diffusion losses. It is a combination of a resistance $R c t$ for the charge transfer resistance and a nonlinear Warburg impedance $Z_{w}$ adopted to reproduce the effects of the mass transfer i.e. water and gas diffusion. This value will reflect issues linked to water and gas management, such as flooding, oxygen and hydrogen starvation, etc. Any variation of $R_{c t}$ and $Z_{w}$ indicates a variation of the activation loss and diffusion loss due to i.e. PTFE/carbon composition at GDL and/or Pt catalyst dissolution at AL. The double layer capacitor $C_{d l}$ explains the porous electrodes effect, i.e. inhomogeneous electrode surface or relaxation processes. 


\section{Degradation Measurements for Battery and PEFMC}

\subsection{State Of Charge (SOC) and State Of Health (SOH)}

The SOC and $\mathrm{SOH}$ are indicators that are commonly used for batteries. Different methods are proposed to define these indicators. One way of defining SOC and $\mathrm{SOH}$ is given in Figure 6. Compared to battery SOC variation, battery SOH typically change much more slowly. In 2001, Piller et al. [19] proposed an overview of the methods for SOC determination and linked their ability to also determine $\mathrm{SOH}$. Some methods are presently more usual than others, and the most common methods for SOC estimation are Open Circuit Voltage (OCV) and capacity fading. Moreover, EIS is usually considered the best way to determine the SOH. Contrary to batteries, SOC does not exist for fuel cells. Since PEM fuel cells are continuously fed by external gaseous, it is considered being always fully charged. Subsequently, its 'capacity state' does not exist neither, or at least it does not have the same meaning. It might be equivalent to the external fuel (hydrogen) storage. However this 'capacity' does not represent the PEMFC ageing. Nevertheless, considering the gases and water management inside the cell, could be interesting to introduce the State of Supply (SoS). The health issues of SoS thus can be owing to gas starvation. As for Li-ion battery, $\mathrm{SOH}$ has no unique definition since it does not correspond to a particular physical quality.

The wider experience in batteries will be used as a basis to discuss about the fuel cell $\mathrm{SOH}$ determination, and therefore OCV, capacity fading and EIS will be considered in the sequel.

\subsection{Open Circuit Voltage (OCV)}

The battery OCV gives information about its capacity at current charge status, and is therefore widely used for both SOC and SOH estimations [11, 20, 21]. In [21], a reading of the battery OCV is converted to SOC by using an ECM. A look-up table of OCV-SOC is usually built by an experimental approach. $\mathrm{OCV}$ is a common measurement for battery but for PEMFC, the 
OCV measurement can cause severe damage of the fuel cell stack if it takes too long time. Chemical degradation caused by reactant (hydrogen or oxygen) crossover is most severe under OCV conditions. This may lead to the formation of peroxide species, which will accelerate the degradation of the electrolyte membrane and lead to failure of the MEA by compromising the integrity of the electrolyte membrane [21]. For these reasons, this method is avoided. However many studies use it, and in normal operation, some "accidental" transient crossing of OCV can still be exploited.

\subsection{Capacity}

Capacity is rated in Ampere-hours (Ah) quantifying the available energy stored in a battery. The loss of capacity results in increased impedance and reduced performance. The rate of capacity loss is highly dependent on the operating conditions such as maximum charge voltage, depth of discharge, magnitude of current, load profiles and temperature. The traditional charge/discharge/charge cycle still offers a dependable way to measure battery capacity $[2,22-23]$. But the process is time-consuming and it needs to remove the battery from service for the duration of the test. Thus, on-board capacity estimation methods are searched [2]. When the battery is being charged or discharged, its voltage generally increases or decreases, respectively. The discharging or charging of a certain amount of Ampere-hours creates a higher voltage change for a battery with a lower capacity than that for a battery of the same type but with a higher capacity. Therefore, the battery capacity can be considered as a parameter defining the relationship between the Ampere-hours charged or discharged from the battery and voltage difference before and after the respective charging or discharging. The determination of this relationship is therefore the basic principle of almost all methods for onboard capacity estimation [2]. Those studies on battery capacity fading are based on measurements such as OCV curves. 
The performance of Li-ion battery can also be expressed in term of Watt-hours which is the unit of energy measurement indicating how much energy can be drawn from the battery for a certain number of hours [13]. Thus, power fade becomes a more meaningful indicator of battery performance in applications such as hybrid electric vehicles. Theoretically, Watt-hour and Ampere-hour can be converted to each other via the formula:

$W h=A h^{*} V$

As a result, power fade modeling and prediction can utilize the same functional forms as used for the capacity fade models. In the respect of storage, the PEMFC is not working as an energy pool as Li-ion battery but an online power converter, and the capacity has thus no meaning. PEMFCs indicator is usually the stack power, which, according to the above section, leads to the same information as the capacity.

\subsection{EIS and ECM for SOH determination}

Figure 7 shows the typical trend of the Impedance Spectroscopy of a lithium battery when it is fully charged [24]. It highlights the ageing spectroscopy comparison between experimental Impedance Spectroscopy and the equivalent circuit model results at different cycle ageing. Except the first cycle of activation, the total module of the impedance enhances with the ageing. In [24], the parameters of the model (Figure 8) are reported versus the SOC and at different number of cycles. Among the different parameters, the ohmic resistance of the battery enhances during the ageing, but it remains mostly constant during the discharge cycle. Excluding the ageing effects related to the temperature, the enhancement of the ohmic resistance with the ageing is probably due to a growth of the SEI on the anode side of the battery, and it is one of the principal reasons of the lithium battery failure. The trends of the other parameters are not completely clear and it is difficult to use them to predict SOC or $\mathrm{SOH}$. The evolution of the PEMFC EIS diagram at different time stages is shown in Figure 9 with data taken in [25]. The two semi-circles are represented by a parallel connection of a 
resistance and a constant phase element (CPE), which is serially connected to a resistance for ohmic resistance. Two inductances are introduced in parallel and in serial, respectively, to describe the inductive behaviors respectively at high and low frequency.

Kim et al. [25] reported that the low frequency inductance is usually influenced by water transport at the anode and possible carbon monoxide poisoning, while other researchers invoked relaxation of adsorbed intermediates species associated with cathodic reactions. The high frequency inductive part, on the other hand, is usually associated to connecting element and wires.

The ECM model, represented in Figure 10, has the following parameters: the resistances $R_{e l}$, $R_{1}, R_{2}$, and $R_{3}$; the Changing Phase capacitances $C_{1}, C_{2}$ and the inductances $L_{1}, L_{2}$. According to Kim et al [25], some parameters remain unchanged with ageing (capacitances and inductances) while others (resistances) seem to be sensitive to ageing. Thus, the authors considered $R_{e l}, R_{l}, R_{2}$, and $R_{3}$ as primary parameters linked to ageing. Besides the different resistances, Onanéna et al. [26] selected other parameters to describe the evolution of the EIS diagrams with ageing: the minimum value of the imaginary part in the impedance spectrum, its corresponding real part values and its occurring frequency (3 parameters in total).

\section{Towards a specific PHM approach: Ageing Indicators}

In the previous parts, the ageing mechanisms, characterization tools and some degradation measurements for SOC and SOH determination have been explored for both batteries and fuel cells. This part discusses now how to build a relevant ageing indicator or SOH indicator based on the present knowledge. Thus, the best way to combine the characterization tools and degradation measurements is studied, so as to supervise the most significant physical degradations of the devices. 


\subsection{Covariates Selection and Ageing Indicators for Batteries}

Some research studies consider that the li-ion battery indicator is capacity, for which the fade mechanisms are usually dominated by a unique ageing mechanism - lithium loss due to solid electrolyte interphase (SEI) decomposition. But this approach is greatly simplified and many other studies explore more accurate approaches.

As the deteriorations may have several origins, the use of more than one measurement to identify the different origins is needed. For example, the internal resistance and the capacity can be used as a basis for providing an indication of the battery $\mathrm{SOH}$. The deviation in the battery performance may be due to a sudden rise in temperature throughout the operation; or internal degradations [11]. It is also possible to take account of the environment. In most real in-field applications, the most effective and simple method of characterizing battery behavior is based on measuring battery voltage, current, temperature, and in some cases pressure. For example, Wang et al. [27], proposed a method which takes into account the process of lithium-ion battery capacity fading, battery performance metrics (current, voltage, time, battery temperature, and ambient temperature) and correlated them with operation time.

The use of models allows estimating parameters and their evolution with degradations. Some physics-based models have been developed. They are derived from conservation laws applied to the physics occurring within a Lithium-ion battery, as well as Butler-Volmer kinetic expressions to model reactions occurring within the electrode affecting overpotential. Such models are only valid under steady-state load conditions, since their voltage response to load changes is too responsive. If these models can build a good fundamental understanding of the electrochemical process, they are still far from being applied in practice due to their complexity [11]. 
Furthermore, models using electrical equivalence like ECM are more often used. However the parameters have no direct physical significance. In addition, the parameters are usually assumed to be constant, but in real applications, they may vary as functions of working conditions and battery usage history [11]. Moreover, the ECM model is not capable of simulating the SOC or the capacity fading due to environment temperature or material degradation.

Walker et al. [28] compared three models in order to identify and predict the health parameters in a PHM approach: a data-driven model, an equivalent circuit model (ECM) and physics-based single particle (SP) model. SP model is derived from conservation laws and Butler-Volmer kinetic expressions. Their relevancy to be used in a prognosis algorithm is then tested - only cell voltage is measured, and the time until the end of discharge voltage is then estimated thanks to the resistive and capacitive parameters of an ECM. Similar performances are obtained by using the three models.

A way to improve the accuracy of the ageing indicator is to combine the ECM model with advanced data-driven prognostic techniques. The use of data-driven prognostic techniques may compensate the lack of accuracy of a simple battery model. Both historical data and online monitoring information of the system can be used to train advanced learning tools for battery performance prediction [11].

Liao et al. [29] proposes also a hybrid approach for lithium-ion cells using an ECM model of the battery. The model parameters change with ageing and fault processes, that can thus be used to track the battery degradation. The batteries internal resistances are identified to assess the current $\mathrm{SOH}$ and to estimate RUL predictions. During the learning stage of the prognosis, the approach is similar to standard prognosis using particle filtering algorithm. However, the prediction stage is different. Indeed, new measurements are provided through a data-driven prediction of the capacity values during each discharge cycle of the battery. This method 
provides the possibility to update the particle weights during the prediction phase, and thus to correct an imperfect degradation model or highly varying measurements at the end of the learning phase. Otherwise, RUL prediction can gives inaccurate results.

\subsection{Covariates Selection and Ageing Indicators for PEM Fuel Cells}

Due to the multi-physic nature of fuel cells, its $\mathrm{SOH}$ is influenced by several interacting parameters. Jouin et al. [4] stated that studying the $\mathrm{SOH}$ and ageing of the PEMFC require the availability of at least one good indicator that represents the evolution of the system. Several candidates have been reported by the authors, like the catalyst area degradation or the rate of hydrogen that crosses over the membrane. However, these parameters cannot be easily measured and their monitoring strongly disturbs the PEMFC operation, exacerbates the degradation and accelerates the ageing. On the other hand, ageing and the majority of degradation mechanisms have a direct impact on the output voltage/power, which is a noninvasive and easy-to-monitor parameter in a PEMFC system. Consequently, voltage is a good candidate to understand and predict the ageing of the PEMFC. The observation of an operating PEMFC voltage signal shows the presence of both irreversible and reversible degradations. The irreversible process is due to permanent degradation (ageing), and the reversible part is due to incidents or characterization actions.

Discriminate between causes is currently an unsolved problem for fuel cells, and is limited by the measurement possibilities and the difficulties of establishing a physical model. Some solutions have been explored to overcome this obstacle for diagnosis purpose, and either using conventional measures [9], combination of EIS and physical model [18] or using innovative ones [30] are still open ways.

Until new approaches are available, EIS is a very interesting characterization tool either for diagnosis or prognostics. As described before, in addition to the voltage or power signal, extracted parameters from EIS, such as resistances are good degradation indicators for fuel 
cells $[25,26]$. Since these measures give different information on the fuel cell state of health, it would be interesting to combine them in a hybrid approach, of the same type as the one proposed for batteries.

\section{Conclusion}

This work investigated what could be transposed from the experience on batteries ageing indicators, to the PEM Fuel Cells. However, a strong limitation of the transposition is due to the much more complex operation of fuel cell because of the fluidic part. For batteries, fault causes remain fairly hierarchical, with a predominance of damage related to the SEI interface. However for fuel cells, the lack of dominant degradation and co-existence of reversible / irreversible damage makes difficult to interpret the measurements and to discriminate between the different causes. The complete knowledge is still lacking for fuel cells. To select the suitable covariates to be used as ageing indicators, the approaches used for batteries propose some specific measurement, such as using the OCV or capacity fading which are hardly transferable to PEMFC. . Nevertheless, a parallel can still be drawn between the two technologies. Thus, the monitoring of the capacity degradation for batteries is similar to monitoring the power degradation for PEMFCs. In addition, both battery and PEMFC SOH can be obtained from the EIS curve. Consequently, hybrid approaches, combining two sources of information that have been proposed for batteries can be transposed to PEMFCs. This study has revealed many limitations of the comparison. It reinforces, however, the research already undertaken for fuel cells as the comparison with batteries methods confirms that the methods explored for PEMFC are relevant. This comparison also reveal that the hybrid approaches that are developed for batteries seems to be more suitable to characterize ageing and build ageing indicators and should be investigated for PEMFC too. 


\section{Acknowledgements}

This work has been performed in cooperation with the Labex ACTION program (contract ANR-11-LABX-0001-01).

\section{References}

[1] Breaz, E.; Fei, G.; Miraoui, A.; Tirnovan, R., IECON 2014 IEEE, 2014, 3941-3947.

[2] Waag, W.; Fleischer, C.; Sauer, D. U. J. Power Sources 2014, 258, 321-339.

[3] Barré, A.; Deguilhem, B.; Grolleau, S.; Gérard, M.; Suard, F.; Riu, D. J. Power Sources 2013, 241, 680-689.

[4] Jouin, M.; Gouriveau, R.; Hissel, D.; Péra, M.-C.; Zerhouni, N. Int. J. Hydrogen Energy 2013, 38 (35), 15307-15317.

[5] Yousfi Steiner, N.; Hissel, D.; Moçotéguy, P.; Candusso, D.; Marra,; Pianese, D.C.; Sorrentino, M., Fuel Cells, 2012, 12(2), 302-309.

[6] Liu, J.; Gazzarri, J.; Eikerling, M., Fuel Cells, 2013, 13(2), 134-142

[7] Li, Zi.; Giurgea, S.; Outbib, R.; Hissel, D., Fuel Cells, 2014, 14(3), 448-456

[8] Gašperin, M.; Boškoski, P.; Debenjak, A.; Petrovčič, J., Fuel Cells, 2014, 14(3), 457465.

[9] Petrone, R.; Zheng, Z.; Hissel, D.; Péra, M. C.; Pianese, C.; Sorrentino, M.; Becherif, M.; Yousfi-Steiner, N. Int. J. Hydrogen Energy 2013, 38 (17), 7077-7091.

[10] Zou, Y.; Hu, X.; Ma, H.; Li, S.E., J. Power Sources 2015, 273, 793-803

[11] Rezvanizaniani, S. M.; Liu, Z.; Chen, Y.; Lee, J. J. Power Sources 2014, 256, 110-124.

[12] Vetter, J.; Novák, P.; Wagner, M. R.; Veit, C.; Möller, K. C.; Besenhard, J. O.; Winter, M.; Wohlfahrt-Mehrens, M.; Vogler, C.; Hammouche, a. J. Power Sources 2005, 147 (1-2), 269-281.

[13] Guo, J.; Li, Z.; Pecht, M. J. Power Sources 2015, 281, 173-184.

[14] Dubau, L.; Castanheira, L.; Maillard, F.; Chatenet, M.; Lottin, O., Wiley Interdiscip. 
Rev. Energy Environ. 2014, 3 (6), 540-560.

[15] Wu, J.; Yuan, X. Z.; Martin, J. J.; Wang, H.; Zhang, J.; Shen, J.; Wu, S.; Merida, W. J. Power Sources 2008, 184 (1), 104-119.

[16] Ehrlich, G. M. Lithium-Ion Batteries; 2002.

[17] Barai, A.; Chouchelamane, G. H.; Guo, Y.; McGordon, A.; Jennings, P. J. Power Sources 2015, 280, 74-80.

[18] Tant, S.; Rosini, S.; Thivel, P.-X.; Druart, F.; Rakotondrainibe, a.; Geneston, T.; Bultel, Y. Electrochim. Acta 2014, 135, 368-379.

[19] Piller, S.; Perrin, M.; Jossen, A. J. Power Sources 2001, 96 (1), 113-120.

[20] Chiang, Y.-H.; Sean, W.-Y.; Ke, J.-C. J. Power Sources 2011, 196 (8), 3921-3932.

[21] He, H.; Zhang, X.; Xiong, R.; Xu, Y.; Guo, H. Energy 2012, 39 (1), 310-318.

[22] Wang, D.; Miao, Q.; Pecht, M. J. Power Sources 2013, 239, 253-264.

[23] Dong, H.; Jin, X.; Lou, Y.; Wang, C. J. Power Sources 2014, 271, 114-123.

[24] Galeotti, M.; Cinà, L.; Giammanco, C.; Cordiner, S.; Di Carlo, A. Energy 2015, 89, $678-686$.

[25] Youn, B. D. 2014 Int. Conf. Progn. Heal. Manag. 2014, 1-7.

[26] Onanena, R.; Oukhellou, L.; Candusso, D.; Harel, F.; Hissel, D.; Aknin, P. Int. J. Hydrogen Energy 2011, 36 (2), 1730-1739.

[27] Wang, S.; Zhao, L.; Su, X.; Ma, P. Energies 2014, 7 (10), 6492-6508.

[28] Walker, E.; Rayman, S.; White, R. E. J. Power Sources 2015, 287, 1-12.

[29] Liao, L.; K??ttig, F. IEEE Trans. Reliab. 2014, 63 (1), 191-207.

[30] Chevalier, S.; Auvity, B.; Olivier, J. C.; Josset, C.; Trichet, D.; Machmoum, M., Fuel Cells, 2014,14(3), 416-429.

[31] Hamaz, T.; Cadet, C.; Druart, F.; Cauffet, G. 19th World Congr. Int. Fed. Autom. Control Cape 2014, 11482-11487. 


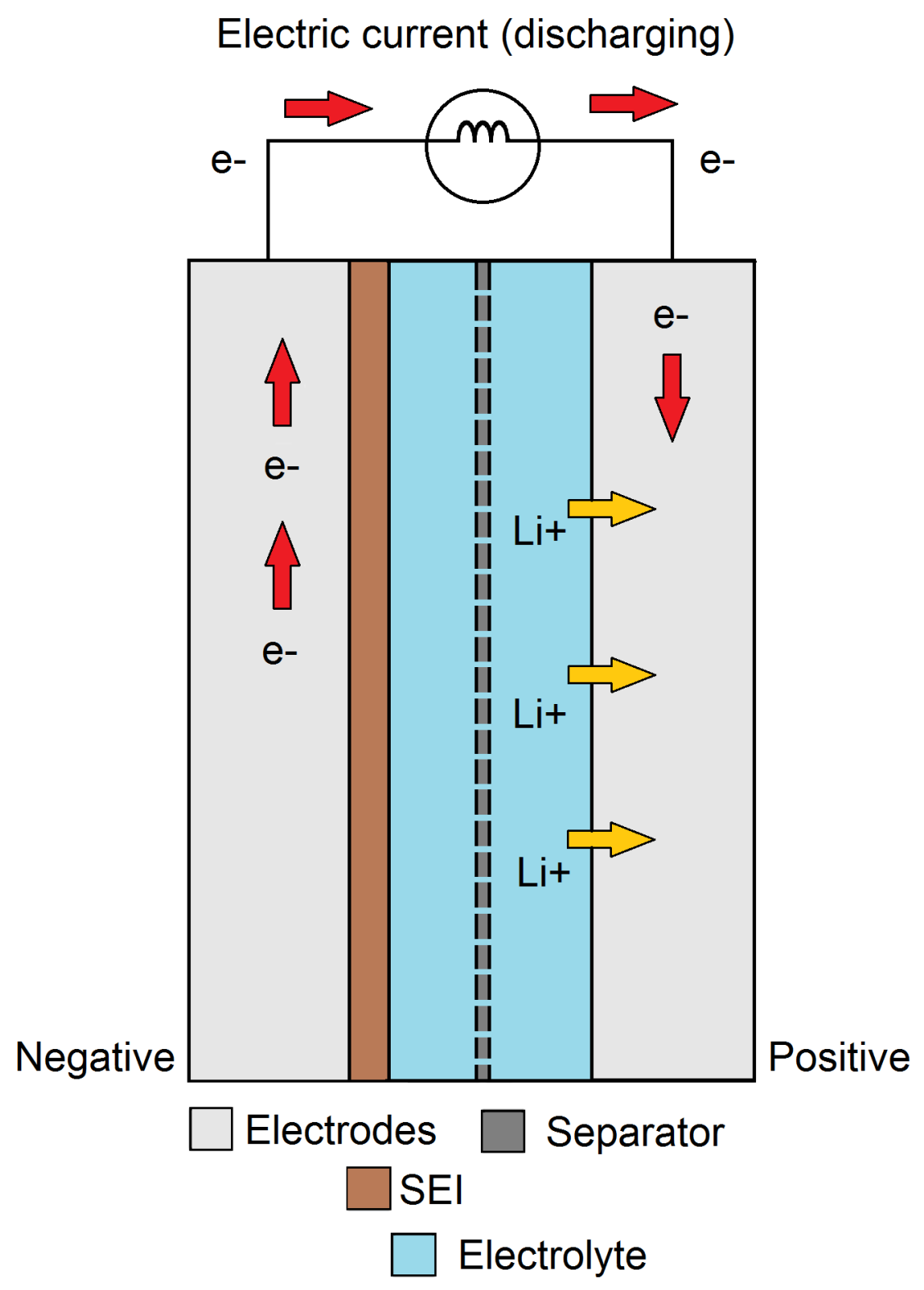

Figure 1. Li-ion battery cell at discharging 


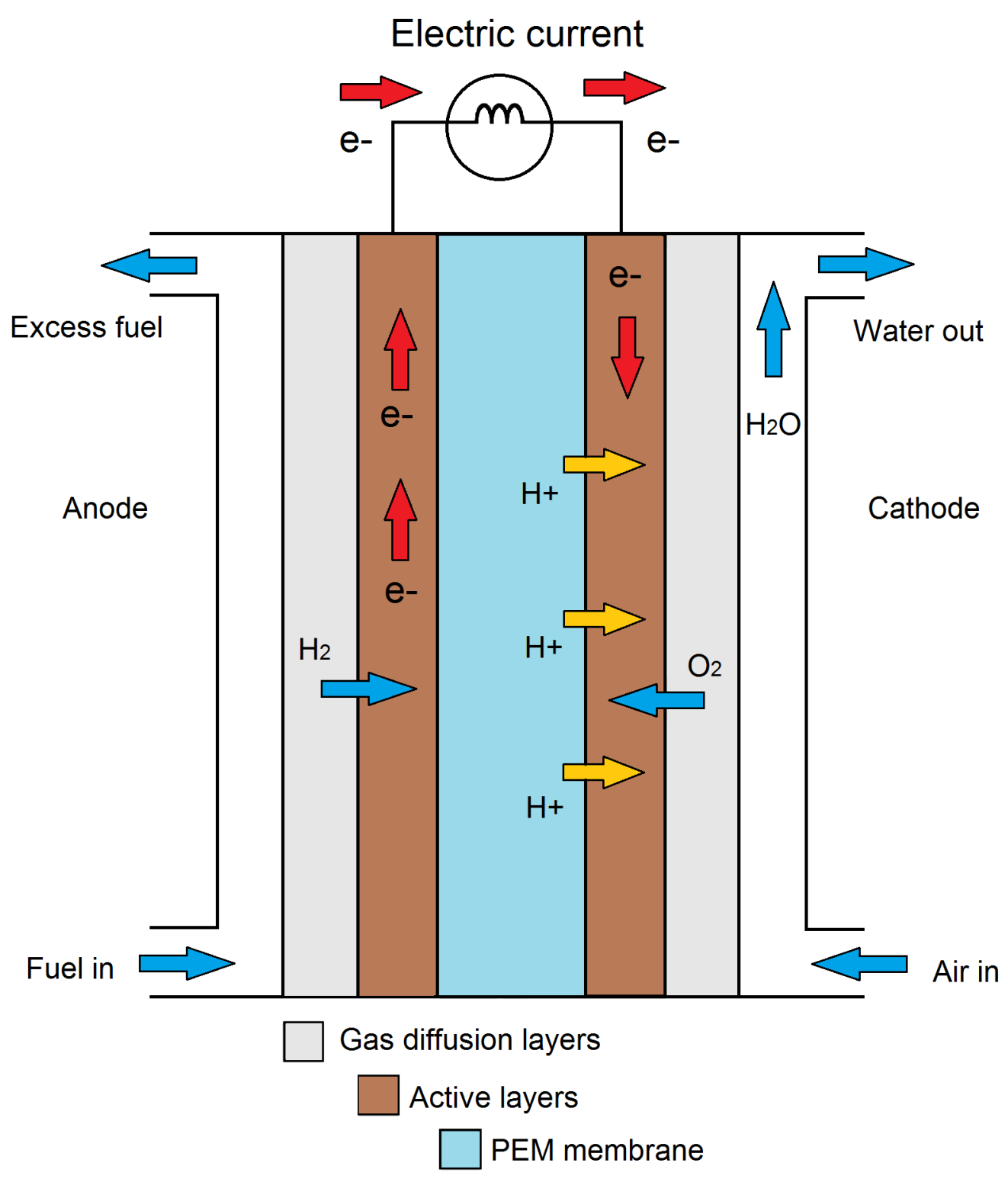

Figure 2. PEMFC 


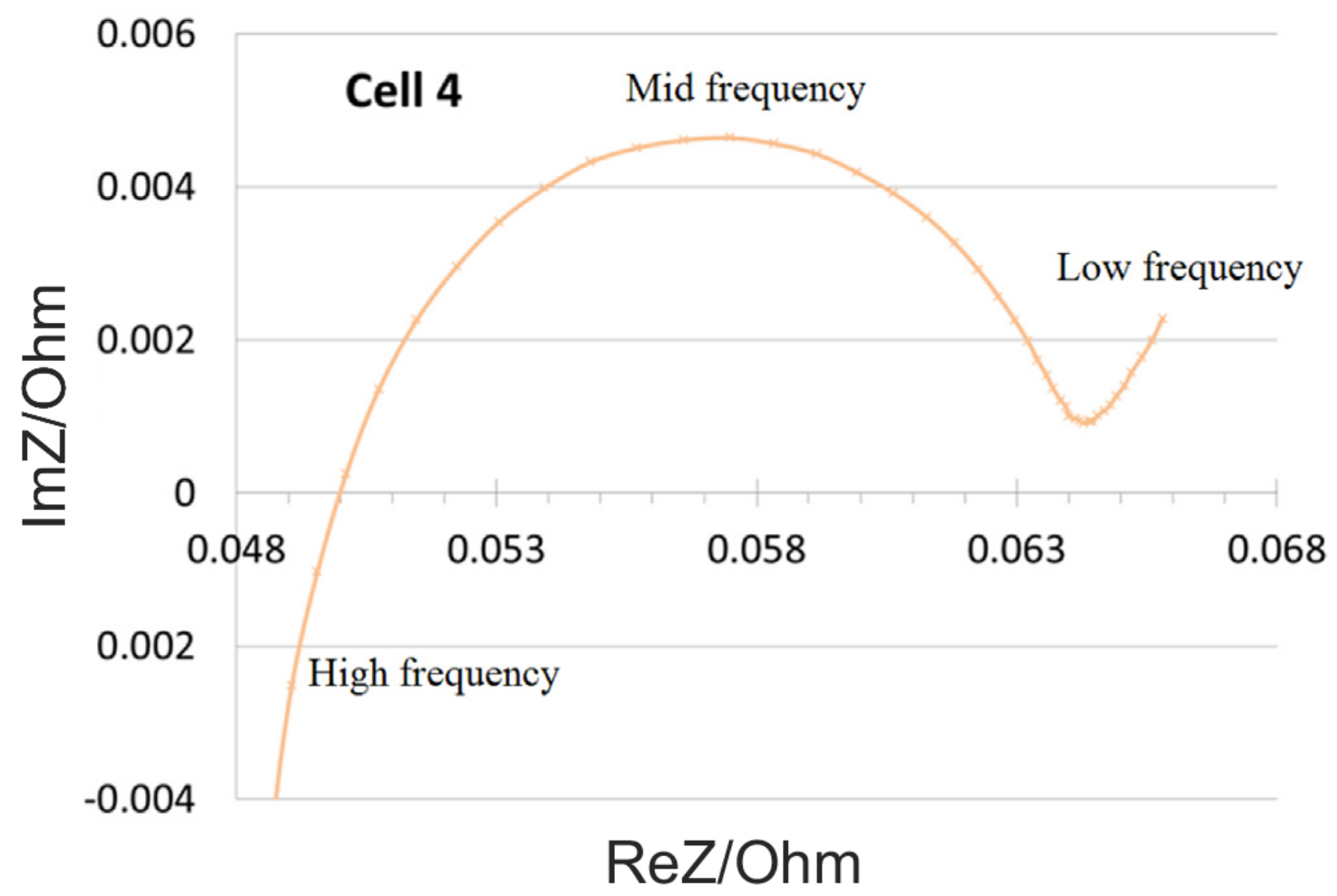

Figure 3. EIS diagram of Li-ion battery [17] 


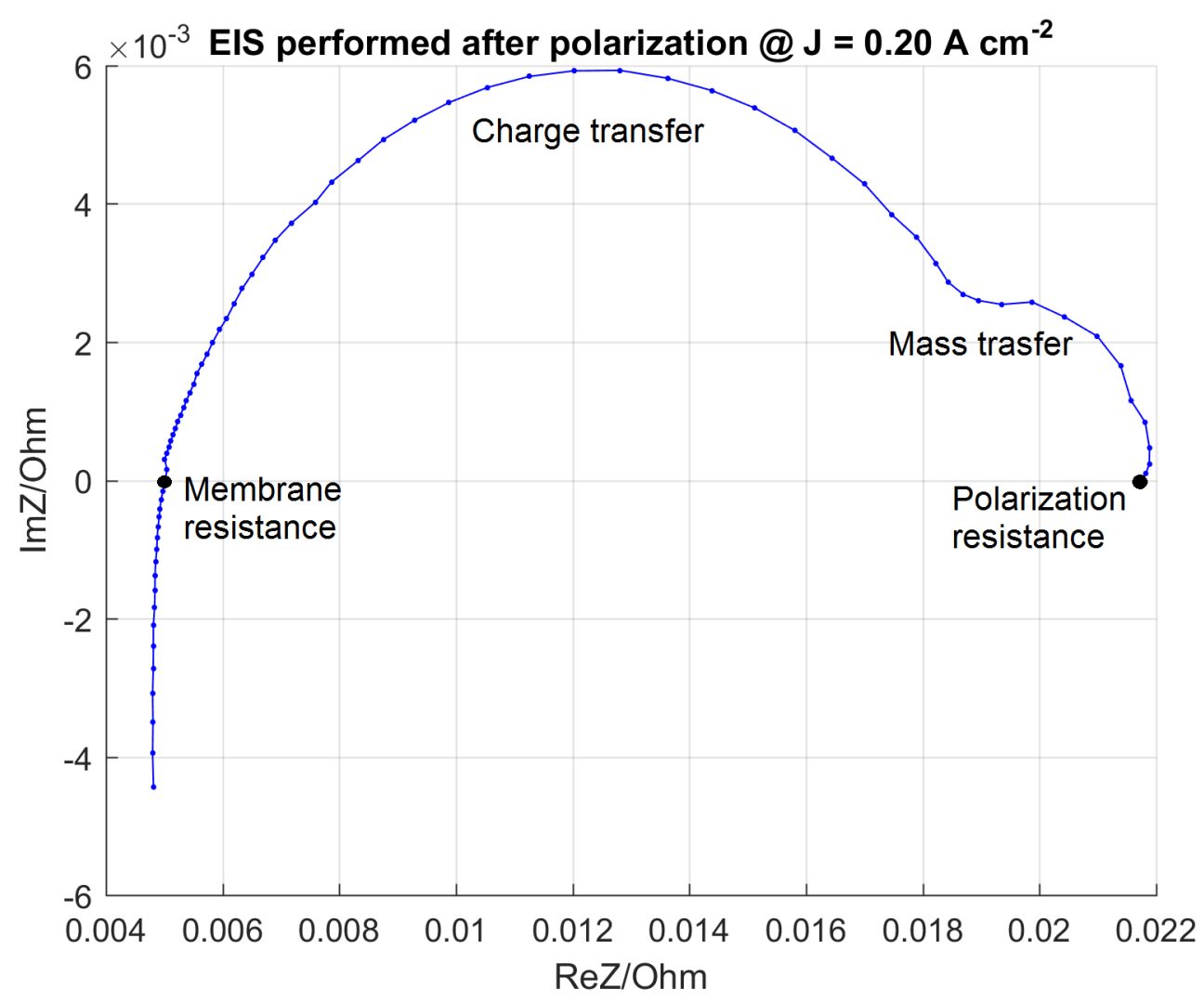

Figure 4. EIS diagram of a Fuel Cell and representation of the different phenomena inside the cell 


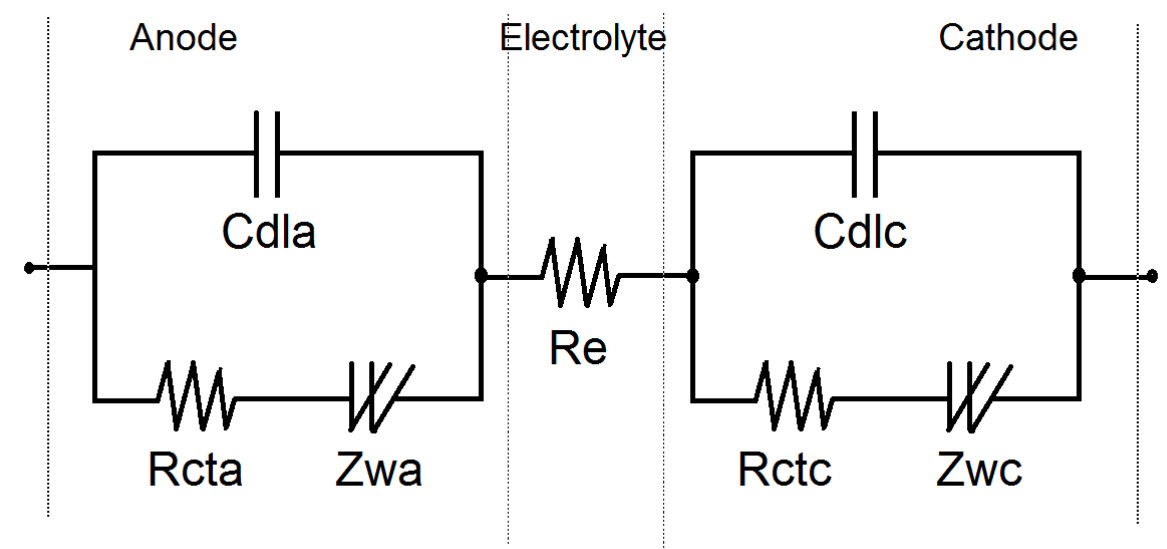

Figure 5. ECM for electrochemical generator 


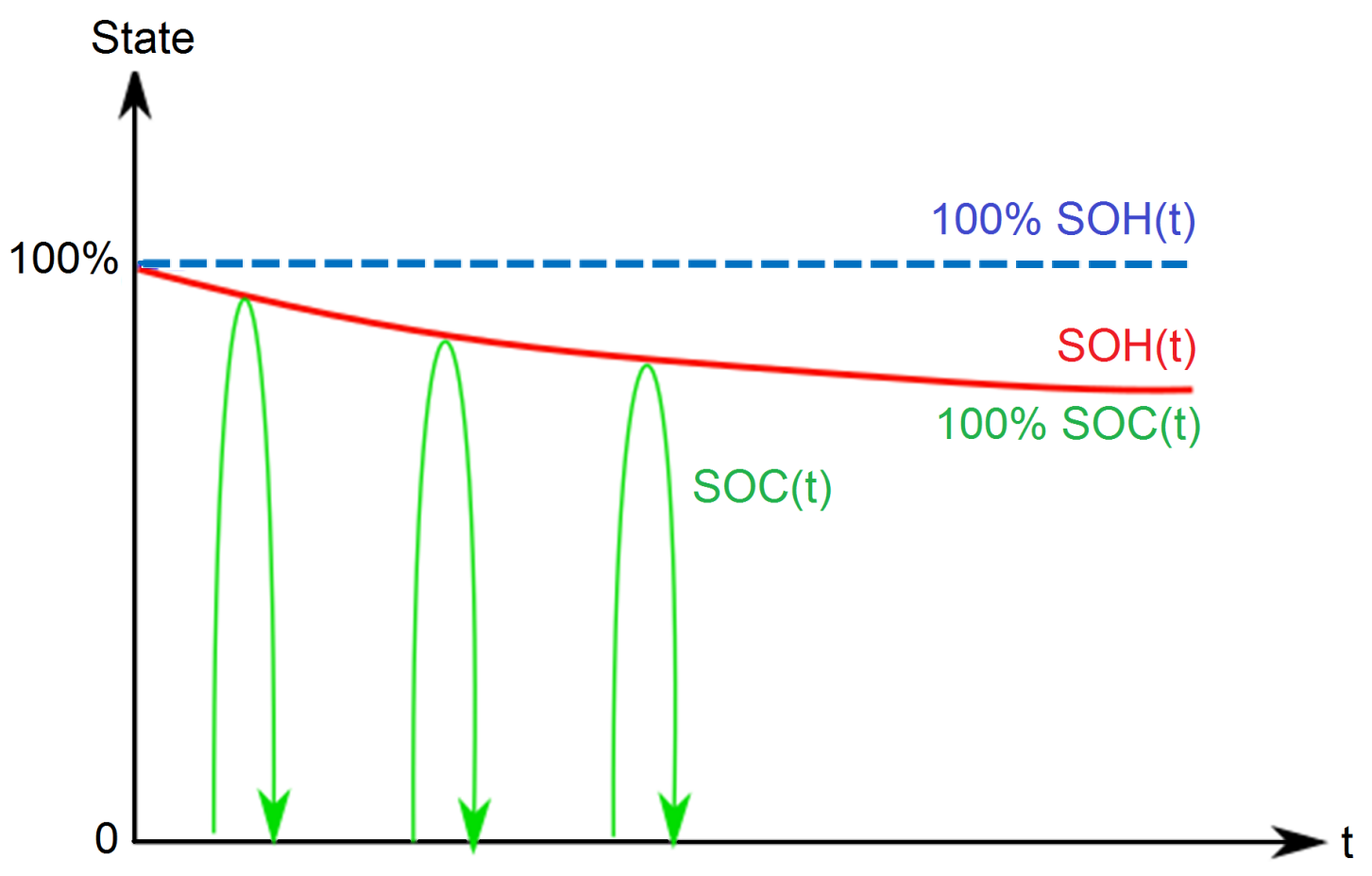

Figure 6. SOC and SOH definition based on battery capacity 


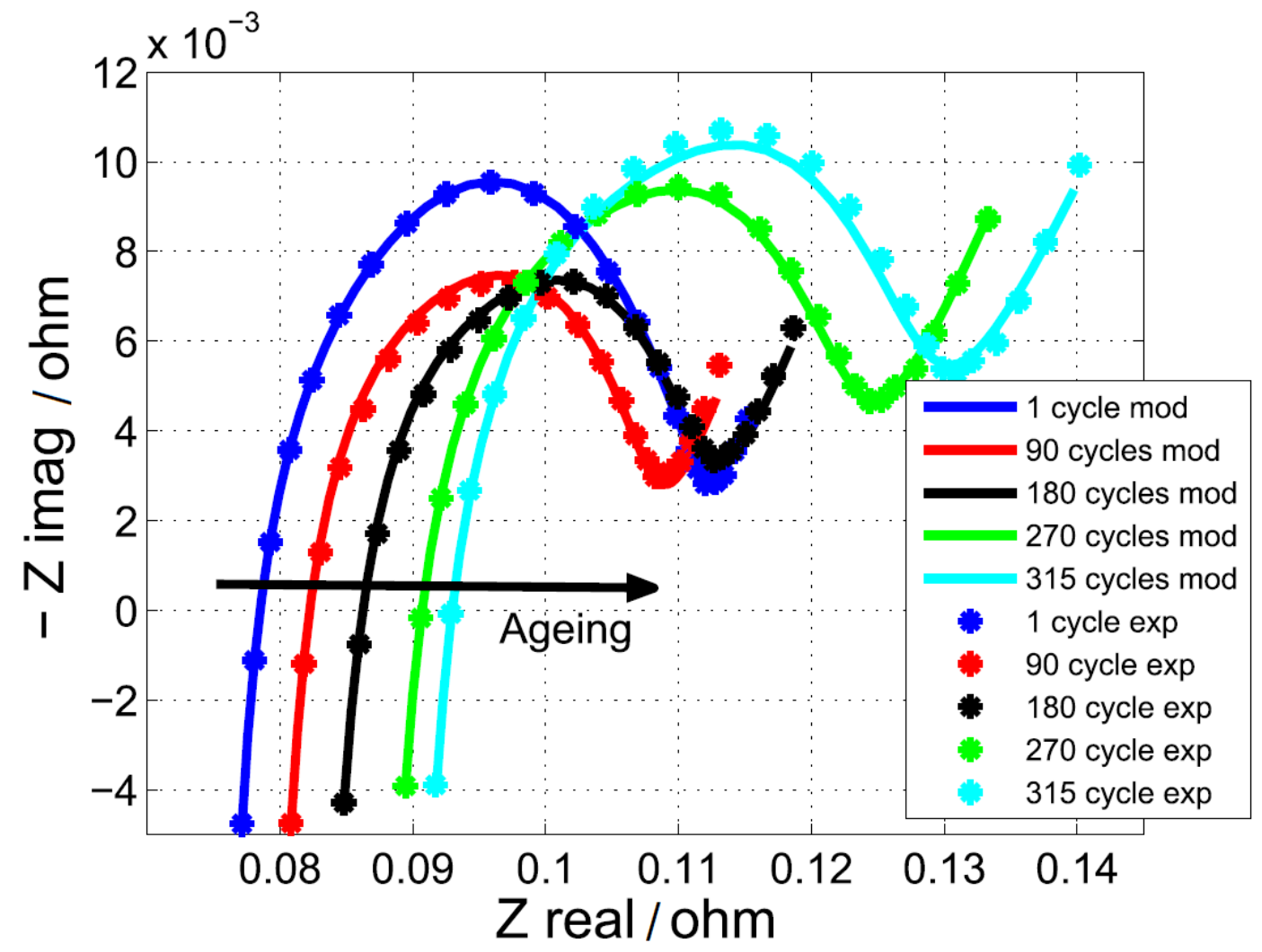

Figure 7. Experimental versus model data. EIS acquired at different number of cycles (operating temperature $25^{\circ} \mathrm{C}$ ) [24] 


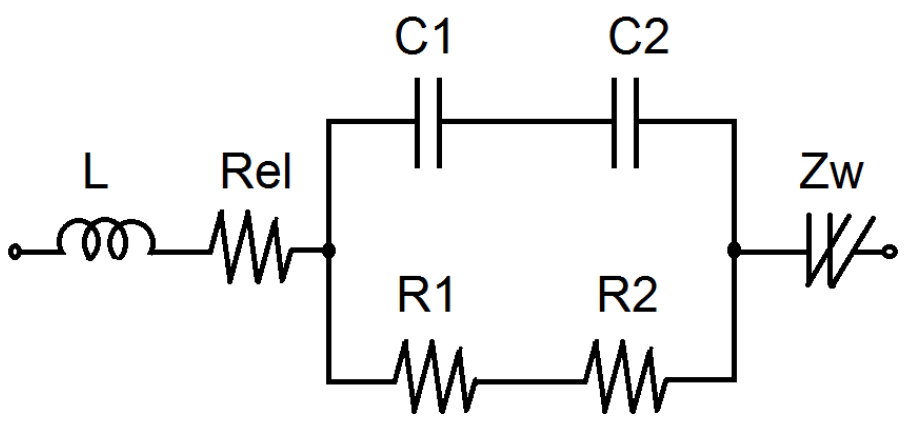

Figure 8 . Battery equivalent circuit 


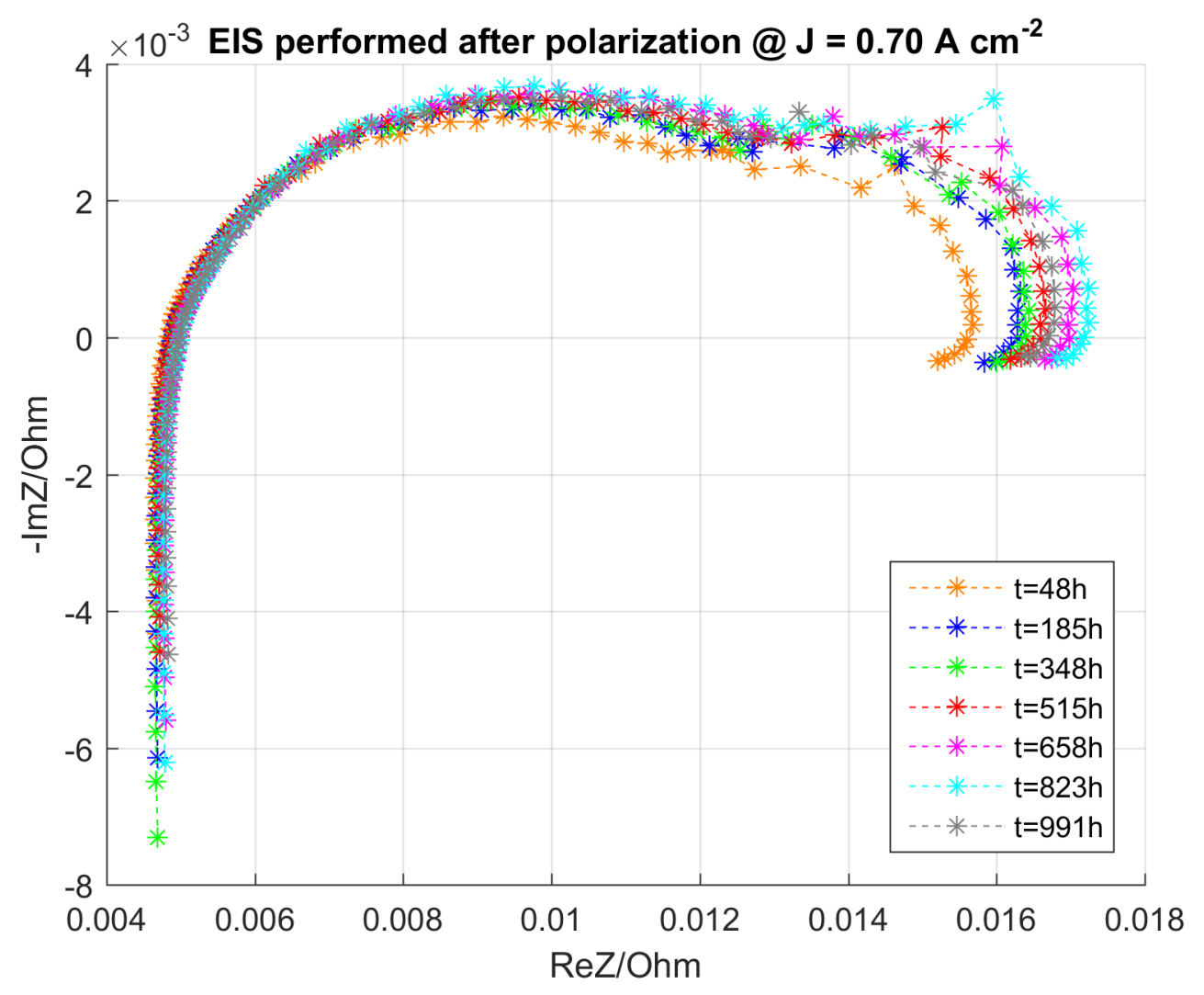

Figure 9. PEMFC EIS at different time stage 


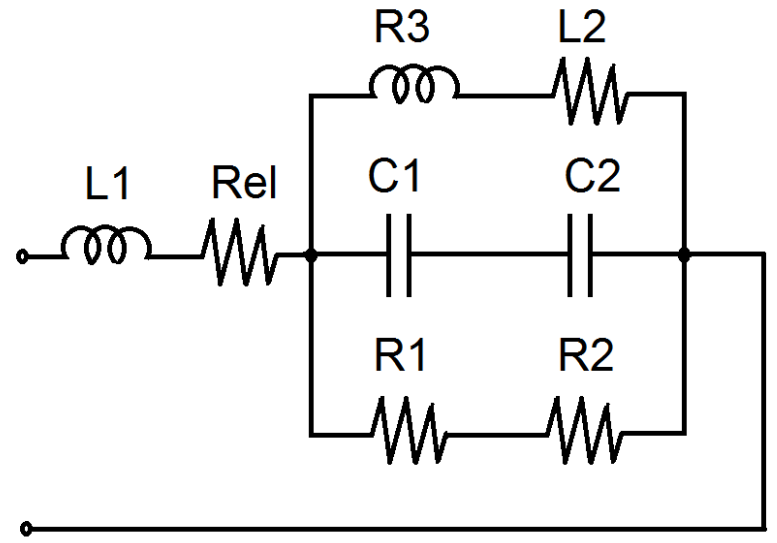

Figure 10. PEMFC ECM circuit 